\title{
Control of oscillating foil for propulsion of biorobotic autonomous underwater vehicle (AUV)
}

doi:10.1533/abbi.2004.0044

\author{
S. N. Singh and S. Mani \\ Department of Electrical and Computer Engineering, University of Nevada, Las Vegas, NV 89154-4026, USA
}

\begin{abstract}
The paper treats the question of control of a laterally and rotationally oscillating hydrofoil for the propulsion of biologically inspired robotic (biorobotic) autonomous underwater vehicles (BAUVs). Sinusoidal oscillations of foils produce maneuvering and propulsive forces. The design is based on the internal model principle. Two springs are used to transmit forces from the actuators to the foil. Oscillating fins produce periodic forces, which can be used for fish-like propulsion and control of autonomous underwater vehicles (AUVs). The equations of motion of the foil include hydrodynamic lift and moment based on linear, unsteady, aerodynamic theory. A control law is derived for the lateral and rotational sinusoidal oscillation of the foil. In the closed-loop system, the lateral displacement and the rotational angle of the foil asymptotically follow sinusoidal trajectories of distinct frequencies and amplitudes independently. Simulation results are presented to show the trajectory tracking performance of the foil for different freestream velocities and sinusoidal command trajectories.
\end{abstract}

Key words: Biorobotic AUV, hydrofoil oscillation control, servoregulator.

\section{INTRODUCTION}

Presently, there is considerable interest in the development of biorobotic autonomous underwater vehicles (BAUVs) which can propel and maneuver themselves like marine animals. Aquatic animals are excellent swimmers and have ability to perform intricate maneuvers using flapping tails and fins. Fishes use a variety of fins (dorsal, caudal, pectoral, pelvic fins, etc.) for maneuvering and propulsion (Azuma 1992, Sfakiotakis et al 1999). Studies from fish and cetaceans has inspired researches to use oscillatory foils to produce propulsive and maneuvering forces for the control of AUVs (Triantafyllou and Triantafyllou 1995, Harper et al 1998, Bandyopadhyay et al 1999a, 1999b, Kato 2002, Lee et al 2003). Readers may refer to a special issue of IEEE Journal of Oceanic Engineering, which has several research papers on biologically inspired science and technology (Bandyopadhyay 2002, Lauder and Drucker 2004, Triantafyllou et al 2004, Walker 2004). The forces and moments produced by the oscillating hydrofoils are

Corresponding Author:

S. N. Singh

Department of Electrical and Computer Engineering

University of Nevada

Las Vegas, NV 89154-4026, USA

Tel: (702) 895-3417; Fax: (702) 895-4075

Email: sahaj@ee.unlv.edu complicated functions of mode of foil oscillation (lead-lag, feathering, and flapping motion) as well as the oscillation parameters (amplitude and frequency of oscillation, bias and phase angle). Fluid dynamicists are involved in using experimental and computational methods to characterize the forces and moments produced by oscillating foils (Triantafyllou and Triantafyllou 1995, Bandyopadhyay et al 1999a, Lee et al 2003, Mittal 2004, Singh et al 2004). Although these results are extremely useful, it is especially important to develop analytical models of lift and moment obtainable from foil oscillation for the application of control system design for maneuvering AUVs.

In an interesting work, motivated by the work of Lighthill (1970), Harper et al (1998) have developed a low-order model of a two-dimensional (2-D) oscillating foil based on the theory of unsteady aerodynamics, which has its origins in the work of Theodorsen (1935). The foil has lateral and rotational motion and represents reasonably the typical tail of thunniform swimmers which have high aspect ratio. Furthermore, in the model of Harper et al, springs are attached to the foil, and forces and moments are transmitted to the foil (tail) by the springs from the actuators. These springs are analogous to fish tendons, which store energy while transmitting forces from muscles.

The forces and moments are functions of oscillating parameters including frequencies of oscillations, phase angles and biases of the foil executing lateral and angular motion. Thus, for generating propulsive and control forces so that 
the AUV can accomplish specific maneuvers, it is essential to control the flapping and pitching motion of the foil. However, by a simple application of actuating signals to the springs by the actuators, one cannot force the foil to oscillate with desirable oscillation parameters due to the coupled lateral and rotational dynamics. Therefore, there is a need to design control system so that the foil has any specified pattern of oscillatory motion and the lateral and rotational displacements are independent of each other.

The contribution of this paper lies in the design of a control system for the independent asymptotic control of the lateral and rotational motion of a 2-D hydrofoil based on the internal model principle (servomechanism theory) (Davison 1976, Wonham 1985). The foil is spring driven by two actuating signals and it experiences lateral displacement and the angular rotation in the free stream. The foil model includes hydrodynamic forces computed using the theory of unsteady aerodynamics. A command generator is used to generate specified command trajectories, which are linear combinations of sinusoidal functions of distinct frequencies, amplitudes, phase angles and average values. A feedback control law is designed so that plunge displacement and pitch angle of the foil asymptotically tracks the command trajectories generated by the command generator. The control system includes a servocompensator, which is fed by the lateral and rotational trajectory errors. Since the states associated with the Theodorsen function cannot be measured, an observer is designed to obtain the estimates of the unavailable states. Then the controller is synthesized using the estimated state variables. Simulation results are presented which show that in the closed-loop system, independent asymptotic control of the plunge displacement and pitch angle trajectories are accomplished.

The presentation of this paper is as follows. Section "Mathematical model" presents the mathematical model. A state variable representation of the hydrofoil model is obtained in Section "State variable representation". The design of the controller and the observer are considered in Section "Control law" and Section "Observer design", respectively, and simulation results are presented in Section "Simulation results".

\section{MATHEMATICAL MODEL}

The spring-driven hydrofoil including a lateral spring is shown in Figure 1. $L$ and $M$ are the hydrodynamic lift and moment and $F_{\mathrm{a}}$ and $\tau_{\mathrm{a}}$ are the driving force and torque applied to the foil at the axis of rotation by the lateral and rotational springs controlled by two independent actuators. (The rotational spring is not shown in the figure.) The complete equations of motion of the foil based on the unsteady aerodynamic theory has been derived in Harper et al (1998), which are given by

$$
\begin{aligned}
& m\left(\ddot{z}_{\mathrm{t}}+\ddot{\theta}_{\mathrm{t}} b\right)=L+F_{\mathrm{a}} \\
& \mathcal{F} \ddot{\theta}_{\mathrm{t}}=M+\tau_{\mathrm{a}}-F_{\mathrm{a}} b
\end{aligned}
$$

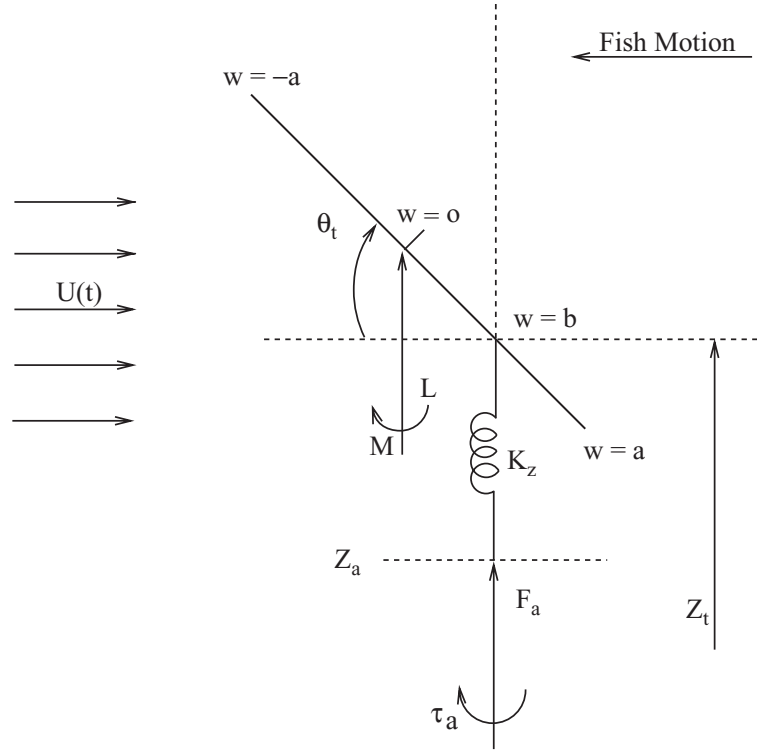

Figure 1 Spring-driven hydrofoil.

where $z_{\mathrm{t}}$ is the vertical position (plunge displacement) and $\theta_{\mathrm{t}}$ is the angular position (pitch angle) of the foil, $m$ is the mass, $\mathcal{F}$ is the moment of inertia, and $b$ is the position of the axis of rotation along the chord.

A complete derivation of the hydrodynamic lift and moment including the added mass and wake effect based on unsteady aerodynamic theory has been obtained in Harper et al (1998). The lift and moment are given by

$$
\begin{aligned}
L= & \pi \rho\left[2 a U\left(-\dot{z}_{\mathrm{t}}+U \theta_{\mathrm{t}}+\left(\frac{a}{2}-b\right) \dot{\theta}_{\mathrm{t}}\right) C(\mathrm{i} \omega)\right. \\
& \left.+a^{2}\left(-\dot{z}_{\mathrm{t}}+U \dot{\theta}_{\mathrm{t}}-b \ddot{\theta}_{\mathrm{t}}\right)\right] \\
M= & -2 \pi \rho a U\left(\frac{a^{2}}{4}\right) \dot{\theta}_{\mathrm{t}}+\pi \rho a^{2} U\left(-\dot{z}_{\mathrm{t}}+U \theta_{\mathrm{t}}\right. \\
& \left.+\left(\frac{a}{2}-b\right) \dot{\theta}_{\mathrm{t}}\right) C(\mathrm{i} \omega)-\frac{\pi}{8} \rho a^{4} \ddot{\theta}_{\mathrm{t}}
\end{aligned}
$$

where $\rho$ is the density, $a$ is the half chord length of the tail, $U$ is free stream velocity (or equivalently fish's forward motion), and $C(\mathrm{i} \omega)$ is the theodorsen function. A thirdorder transfer function to obtain a good approximation of the Theodorsen function used in the study is

$$
C(\mathrm{i} \omega)=\frac{a_{3}(\mathrm{i} \sigma)^{3}+a_{2}(\mathrm{i} \sigma)^{2}+a_{1}(\mathrm{i} \sigma)+a_{0}}{(\mathrm{i} \sigma)^{3}+b_{2}(\mathrm{i} \sigma)^{2}+b_{1}(\sigma)+b_{0}}
$$

where $\sigma=\frac{\omega a}{U}$ is the non-dimensional reduced frequency and $a_{i}$ and $b_{i}$ are given by

$$
\begin{gathered}
{\left[a_{3}, a_{2}, a_{1}, a_{0}\right]=[0.500000,1.07610,} \\
0.524855,0.045133] \\
{\left[b_{2}, b_{1}, b_{0}\right]=[1.90221,0.699129,0.0455035]}
\end{gathered}
$$

The force and moment, $F_{\mathrm{a}}$ and $\tau_{\mathrm{a}}$ applied to the foil by springs in series with the actuators are

$$
\begin{aligned}
F_{\mathrm{a}} & =K_{z}\left(z_{\mathrm{a}}-z_{\mathrm{t}}\right) \\
\tau_{\mathrm{a}} & =K_{\theta}\left(\theta_{\mathrm{a}}-\theta_{\mathrm{t}}\right)
\end{aligned}
$$


where $K_{z}$ and $K_{\theta}$ are spring constants, and $z_{\mathrm{a}}$ and $\theta_{\mathrm{a}}$ are the positions of the lateral and rotational actuators. According to biomechanists, fish have compliances in their tail tendons to reduce energy costs of muscles. Harper et al (1998) have shown that these springs can similarly reduce actuator energy. Oscillating foil produces periodic forces and moments, which can be utilized for the propulsion and control of AUVs.

Suppose that

$$
Y_{\mathrm{r}}(t)=\left[\begin{array}{c}
z_{\mathrm{r}}(t) \\
\theta_{\mathrm{r}}(t)
\end{array}\right]
$$

is the specified reference trajectory. These trajectories are generated by exosystems given by

$$
\begin{aligned}
& \Pi_{z}(s) \hat{z}_{\mathrm{r}}(s)=0 \\
& \Pi_{\theta}(s) \hat{\theta}_{\mathrm{r}}(s)=0
\end{aligned}
$$

where $\hat{z}_{\mathrm{r}}$ and $\hat{\theta}_{\mathrm{r}}$ denote Laplace transforms of $z_{\mathrm{r}}(t)$ and $\theta_{\mathrm{r}}(t)$, respectively, and $\Pi_{z}(s)$ and $\Pi_{\theta}(s)$ are appropriate polynomials of the form:

$$
\begin{aligned}
& \Pi_{z}(s)=s \Pi_{i=1}^{m_{z}}\left(s^{2}+\omega_{z i}^{2}\right) \\
& \Pi_{\theta}(s)=s \Pi_{i=1}^{m_{\theta}}\left(s^{2}+\omega_{\theta i}^{2}\right)
\end{aligned}
$$

In these polynomials, $\omega_{z i}$ and $\omega_{\theta i}$ are positive real numbers.

We are interested in deriving a control law $U_{\mathrm{c}}=$ $\left(z_{\mathrm{a}}, \theta_{\mathrm{a}}\right)^{\mathrm{T}}$ such that in the closed-loop system the output vector $Y=\left(z_{\mathrm{t}}, \theta_{\mathrm{t}}\right)^{\mathrm{T}}$ asymptotically follows the reference trajectory $Y_{\mathrm{r}}(t)$, that is, the tracking error $\tilde{Y}=$ $\left[\left(z_{\mathrm{r}}-z_{\mathrm{t}}\right),\left(\theta_{\mathrm{r}}-\theta_{\mathrm{t}}\right)\right]^{\mathrm{T}}$ converges to zero as $t \rightarrow \infty$. Note that by the choice of the initial conditions $z_{\mathrm{r} i}^{(i)}(0)$, where $i=0,1,2,3, \ldots\left(m_{z}-1\right), \theta_{\mathrm{r} j}^{j}(0)$, where $j=$ $0,1,2, \ldots\left(m_{\theta}-1\right)$ and frequencies $\omega_{z i}, \omega_{\theta j}$, one can generate a linear combination of sinusoidal trajectories of desirable amplitude, phases, biases and frequencies, in order to produce required control force and moment for the propulsion and control of AUV. Presently, there is considerable interest in exploring the relationships among the fin forces and moments and the modes of oscillation (feathering, lead-lag, and flapping motion) and oscillation parameters of the foils, and numerical and experimental results have been obtained.

\section{STATE VARIABLE REPRESENTATION}

For the purpose of control system design, it will be convenient to obtain a state variable representation of the hydrofoil and the reference trajectory generator. The Theodorsen function can be treated as a filter with input:

$$
\begin{aligned}
V_{\mathrm{f}} & =-\dot{z}_{\mathrm{t}}+U \theta_{\mathrm{t}}+\left(\frac{a}{2}-b\right) \dot{\theta}_{\mathrm{t}} \\
& \triangleq C_{\mathrm{f} k}\left[\begin{array}{c}
z_{\mathrm{t}} \\
\theta_{\mathrm{t}}
\end{array}\right]+C_{\mathrm{f} d}\left[\begin{array}{c}
\dot{z}_{\mathrm{t}} \\
\dot{\theta}_{\mathrm{t}}
\end{array}\right]
\end{aligned}
$$

$\left(\triangleq\right.$ denotes equality by definition) and output $Y_{\mathrm{f}}$

$$
\hat{Y}_{\mathrm{f}}(s)=C(s) V_{\mathrm{f}}(s)
$$

where $C(s)$ is obtained by substituting i $\sigma$ by $\frac{a s}{U}$ in $C(\mathrm{i} \omega)$

The transfer function $C(s)$ can be written as

$$
C(s)=h_{\mathrm{c}}+\frac{h_{2} s^{2}+h_{1} s+h_{0}}{s^{3}+p_{2} s^{2}+p_{1} s+p_{0}}
$$

where $h_{\mathrm{c}}=a_{3}$ and $h_{i}$ and $p_{i}$ are appropriate real numbers.

Although it is possible to obtain infinitely many realizations of $C(s)$, one can obtain a minimal realization of dimension 3 in the form:

$$
\begin{aligned}
\dot{x}_{\mathrm{f}} & =\left[\begin{array}{ccc}
0 & 1 & 0 \\
0 & 0 & 1 \\
-p_{0} & -p_{1} & -p_{2}
\end{array}\right] x_{\mathrm{f}}+\left[\begin{array}{l}
0 \\
0 \\
1
\end{array}\right] V_{\mathrm{f}} \\
& =A_{\mathrm{f} 1}\left(\begin{array}{l}
z_{\mathrm{t}} \\
\theta_{\mathrm{t}}
\end{array}\right)+A_{\mathrm{f} 2}\left(\begin{array}{c}
\dot{z}_{\mathrm{t}} \\
\dot{\theta}_{\mathrm{t}}
\end{array}\right)+A_{\mathrm{f} 3 x_{\mathrm{f}}} \\
Y_{\mathrm{f}} & =h_{\mathrm{c}} V_{\mathrm{f}}+h_{0} x_{\mathrm{f} 1}+h_{1} x_{\mathrm{f} 2}+h_{2} x_{\mathrm{f} 3} \\
& =h_{\mathrm{c}} V_{\mathrm{f}}+\left(h_{0} h_{1} h_{2}\right) x_{\mathrm{f}} \\
& =h_{\mathrm{c}} C_{\mathrm{f} k}\left(\begin{array}{c}
z_{\mathrm{t}} \\
\theta_{\mathrm{t}}
\end{array}\right)+C_{\mathrm{f} d}\left(\begin{array}{c}
\dot{z}_{\mathrm{t}} \\
\dot{\theta}_{\mathrm{t}}
\end{array}\right)+C_{\mathrm{f} 2 x_{\mathrm{f}}}
\end{aligned}
$$

where

$$
\begin{aligned}
A_{\mathrm{f} 1} & =\left(\begin{array}{lll}
0 & 0 & 1
\end{array}\right)^{\mathrm{T}} C_{\mathrm{f} k} \\
A_{\mathrm{f} 2} & =\left(\begin{array}{lll}
0 & 0 & 1
\end{array}\right)^{\mathrm{T}} C_{\mathrm{f} d} \\
C_{\mathrm{f} 2} & =\left(\begin{array}{lll}
h_{0} & h_{1} & h_{2}
\end{array}\right)
\end{aligned}
$$

Collecting the coefficients of of $\ddot{z}_{\mathrm{t}}$ and $\ddot{\theta}_{\mathrm{t}}$, one obtains from (1) and (2):

$$
M\left(\begin{array}{c}
\ddot{z}_{\mathrm{t}} \\
\ddot{\theta}_{\mathrm{t}}
\end{array}\right)=D\left(\begin{array}{c}
\dot{z}_{\mathrm{t}} \\
\dot{\theta}_{\mathrm{t}}
\end{array}\right)+K\left(\begin{array}{c}
z_{\mathrm{t}} \\
\theta_{\mathrm{t}}
\end{array}\right)+K_{\mathrm{f}} Y_{\mathrm{f}}+B_{0} U_{\mathrm{c}}
$$

where $U_{\mathrm{c}}=\left(z_{\mathrm{a}}, \theta_{\mathrm{a}}\right)^{\mathrm{T}}$ is the control input,

$$
\begin{aligned}
M & =\left[\begin{array}{cc}
m+\pi \rho a^{2} & m b+\pi \rho a^{2} b \\
0 & \mathcal{J}+\frac{\pi}{8} \rho a^{4}
\end{array}\right], \\
D & =\left[\begin{array}{cc}
0 & \pi \rho a^{2} U \\
0 & -2 \pi \rho \frac{a^{3}}{4} U
\end{array}\right] \\
K & =\left[\begin{array}{cc}
-K_{z} & 0 \\
b K_{z} & -K_{\theta}
\end{array}\right], \quad K_{\mathrm{f}}=\left[\begin{array}{c}
2 \pi \rho a U \\
\pi \rho a^{2} U
\end{array}\right] \\
B_{0} & =\left[\begin{array}{cc}
K_{z} & 0 \\
-b K_{z} & K_{\theta}
\end{array}\right]
\end{aligned}
$$

Substituting for $Y_{\mathrm{f}}$ from (13) and solving for $\left(\ddot{z}_{\mathrm{t}} \ddot{\theta}_{\mathrm{t}}\right)^{\mathrm{T}}$ gives:

$$
\begin{aligned}
& \left(\begin{array}{c}
\ddot{z}_{\mathrm{t}} \\
\ddot{\theta}_{\mathrm{t}}
\end{array}\right)=M^{-1}\left(K+K_{\mathrm{f}} h_{\mathrm{c}} C_{\mathrm{f} k}\right)\left(\begin{array}{c}
z_{\mathrm{t}} \\
\theta_{\mathrm{t}}
\end{array}\right) \\
& +M^{-1}\left(D+K_{\mathrm{f}} h_{\mathrm{c}} C_{\mathrm{f} d}\right)\left(\begin{array}{c}
\dot{z}_{\mathrm{t}} \\
\dot{\theta}_{\mathrm{t}}
\end{array}\right) \\
& +M^{-1} K_{\mathrm{f}} C_{\mathrm{f} 2} x_{\mathrm{f}}+M^{-1} B_{0} U_{\mathrm{c}} \\
& =A_{1}\left(\begin{array}{c}
z_{\mathrm{t}} \\
\theta_{\mathrm{t}}
\end{array}\right)+A_{2}\left(\begin{array}{c}
\dot{z}_{\mathrm{t}} \\
\dot{\theta}_{\mathrm{t}}
\end{array}\right)+A_{3} x_{\mathrm{f}}+B_{1} U_{\mathrm{c}}
\end{aligned}
$$


Define the state vector

$$
x=\left[\begin{array}{lllll}
z_{\mathrm{t}} & \theta_{\mathrm{t}} & \dot{z}_{\mathrm{t}} & \dot{\theta}_{\mathrm{t}} & x_{\mathrm{f}}
\end{array}\right]^{\mathrm{T}} \in \mathfrak{R}^{\mathrm{T}}
$$

A state variable representation of (12) and (15) takes the form:

$$
\begin{aligned}
\dot{x} & =\left[\begin{array}{ccc}
0_{2 \times 2} & I_{2 \times 2} & 0_{2 \times 3} \\
A_{1} & A_{2} & A_{3} \\
A_{\mathrm{f} 1} & A_{\mathrm{f} 2} & A_{\mathrm{f} 3}
\end{array}\right] x+\left[\begin{array}{c}
0_{2 \times 1} \\
B_{1} \\
0_{3 \times 2}
\end{array}\right] U_{\mathrm{c}} \\
& =A x+B U_{\mathrm{c}}
\end{aligned}
$$

where the system matrices are $A \in \mathfrak{R}^{7 \times 7}$ and $B \in \mathfrak{R}^{7 \times 2}$. The output vector $Y$ is then written as

$$
Y=C x=\left[\begin{array}{ll}
I_{2 \times 2} & 0_{2 \times 5}
\end{array}\right] x
$$

\section{CONTROL LAW}

In this section, the state variable model (17) is used for the derivation of a control law such that $y(t)$ asymptotically tracks $y_{\mathrm{r}}(t)$. The derivation of the control systems is based on the internal model principle (Davison 1976, Wonham 1985). The controller includes a servocompensator incorporating the modes of the reference trajectories.

Let $\Pi_{e}(s)$ be a polynomial which is the least common multiple of the two polynomials $\Pi_{z}(s)$ and $\Pi_{\theta}(s)$ and suppose that it has $(2 m+1)$ distinct roots 0 and $\pm j \omega_{i}$, $i=1, \ldots, m$. Then $\Pi_{e}(s)$ takes the form:

$$
\begin{aligned}
\Pi_{e}(s) & =s\left(s^{2}+\omega_{1}^{2}\right) \cdots\left(s^{2}+\omega_{m}^{2}\right) \\
& \triangleq s^{2 m+1}+a_{\mathrm{c} 2 m-1} s^{2 m-1}+\cdots+a_{\mathrm{c} 3} s^{3}+a_{\mathrm{c} 1} s
\end{aligned}
$$

where the coefficients $a_{\mathrm{c} k}$ are defined in (19). Note that $\Pi_{e}(s)$ is an odd polynomial.

For the derivation of control law based on the internal model principle (Davison 1976, Wonham 1985), consider servocompensators driven by the error signals $\left(z_{\mathrm{r}}-z_{\mathrm{t}}\right)$ and $\left(\theta_{\mathrm{r}}-\theta_{\mathrm{t}}\right)$ of the form:

$$
\left(\begin{array}{l}
\hat{Y}_{\mathrm{c} z} \\
\hat{Y}_{\mathrm{c} \theta}
\end{array}\right)=\left[\begin{array}{cc}
\Pi_{e}^{-1}(s) & 0 \\
0 & \Pi_{e}^{-1}(s)
\end{array}\right]\left[\begin{array}{c}
\hat{z}_{\mathrm{r}}(s)-\hat{z}_{\mathrm{t}}(s) \\
\hat{\theta}_{\mathrm{r}}(s)-\hat{\theta}_{\mathrm{t}}(s)
\end{array}\right]
$$

A state variable representation of the system (20) can be easily shown to be

$$
\begin{aligned}
& \dot{x}_{\mathrm{s} z}=A_{\mathrm{s}} x_{\mathrm{s} z}+B_{\mathrm{s}}\left(z_{\mathrm{r}}-c_{1} x\right) \\
& Y_{\mathrm{c} z}=\left(10_{1 \times 2 m}\right) x_{\mathrm{s} z}=c_{\mathrm{s}} x_{\mathrm{s} z} \\
& \dot{x}_{\mathrm{s} \theta}=A_{\mathrm{s}} x_{\mathrm{s} \theta}+B_{\mathrm{s}}\left(\theta_{\mathrm{r}}-c_{2} x\right) \\
& Y_{\mathrm{c} \theta}=c_{\mathrm{s}} x_{\mathrm{s} \theta}
\end{aligned}
$$

where

$$
A_{\mathrm{s}}=\left[\begin{array}{ccccc}
0 & 1 & 0 & \cdots & 0 \\
0 & 0 & 1 & \cdots & 0 \\
\vdots & \vdots & \vdots & \vdots & \vdots \\
0 & -a_{\mathrm{c} 1} & 0 & \cdots & -a_{\mathrm{c} 2 m-1}
\end{array}\right], \quad B_{\mathrm{s}}=\left[\begin{array}{c}
0 \\
0 \\
\vdots \\
1
\end{array}\right]
$$

Define the augmented state vector

$$
x_{\mathrm{a}}=\left(x^{\mathrm{T}}, x_{\mathrm{s} z}^{\mathrm{T}}, x_{\mathrm{s} \theta}^{\mathrm{T}}\right)^{\mathrm{T}} \in \mathfrak{R}^{7+2(2 m+1)}
$$

Then the derivative of $x_{\mathrm{a}}$ can be written as

$$
\begin{aligned}
\dot{x}_{\mathrm{a}} & =\left[\begin{array}{ccc}
A & 0 & 0 \\
-B_{\mathrm{s}} c_{1} & A_{\mathrm{s}} & 0 \\
-B_{\mathrm{s}} c_{2} & 0 & A_{\mathrm{s}}
\end{array}\right] x_{\mathrm{a}}+\left[\begin{array}{c}
B \\
0 \\
0
\end{array}\right] U_{\mathrm{c}}+\left[\begin{array}{cc}
0 & 0 \\
B_{\mathrm{s}} & 0 \\
0 & B_{\mathrm{s}}
\end{array}\right]\left[\begin{array}{c}
z_{\mathrm{r}} \\
\theta_{\mathrm{r}}
\end{array}\right] \\
& =A_{\mathrm{a}} x_{\mathrm{a}}+B_{\mathrm{a}} U_{\mathrm{c}}+B_{d}\left(z_{\mathrm{r}}, \theta_{\mathrm{r}}\right)^{\mathrm{T}}
\end{aligned}
$$

For the asymptotic trajectory tracking of $Y_{\mathrm{r}}(t)$, according to the servomechanism theory (Davison 1976, Wonham 1985), one must find a control law of the form:

$$
U_{\mathrm{c}}=-K_{\mathrm{a}} x_{\mathrm{a}}
$$

such that the closed-loop matrix

$$
A_{\mathrm{ac}}=\left(A_{\mathrm{a}}-B_{\mathrm{a}} K_{\mathrm{a}}\right)
$$

is Hurwitz (i.e., all its eigenvalues have negative real parts). There exists feedback law of the form (24) for stabilization if the transmission zeros of the system (17) and (18) do not coincide with the roots of the polynomials $\Pi_{z}(s)$ and $\Pi_{\theta}(s)$. For the computation of $K_{\mathrm{a}}$, one can use optimal control theory or pole assignment technique. In this study $K_{\mathrm{a}}$ has been obtained assigning eigenvalues of $A_{\mathrm{ac}}$ in a stable region of the complex plane.

\section{OBSERVER DESIGN}

For the synthesis of control law (24), the measurement of the state vector $x$ is essential. However, the state of the filter associated with the Theodorsen function cannot be measured. As such it becomes necessary to obtain an estimate of the state subvector $x_{\mathrm{f}}$.

For the state estimation, consider an observer given by

$$
\dot{\hat{x}}=A \hat{x}+B U_{\mathrm{c}}+F(Y-C \hat{x})
$$

where $\hat{x}$ denotes the estimate of $x$ and $F$ is the feedback matrix. The dynamics of state error $\tilde{x}=x-\hat{x}$ is of the form:

$$
\dot{\tilde{x}}=(A-F C) \tilde{x}=A_{0} \tilde{x}
$$

For the convergence of the state estimation to zero, one selects $F$ such that the eigenvalues of $A_{0}$ are in the left half of the complex plane. Again, one can use the optimal control theory or the pole assignment technique for the computation of $F$. In this study, we have used pole placement design approach for the computation of the feedback matrix $F$.

For the synthesis of control law, the estimated states are substituted in (21) to yield

$$
U_{\mathrm{c}}=-K_{\mathrm{a}}\left[\hat{x}^{\mathrm{T}}, x_{\mathrm{s} z}^{\mathrm{T}}, x_{\mathrm{s} \theta}^{\mathrm{T}}\right]^{\mathrm{T}}
$$

In the closed-loop system, for any reference trajectory $\left(z_{\mathrm{r}}, \theta_{\mathrm{r}}\right)^{\mathrm{T}}$ which satisfies (7), it follows from the servomechanism theory that $\left(z_{\mathrm{t}}(t), \theta_{\mathrm{t}}(t)\right)$ tends to $\left(z_{\mathrm{r}}(t), \theta_{\mathrm{r}}(t)\right)$ as $t \rightarrow \infty$. 

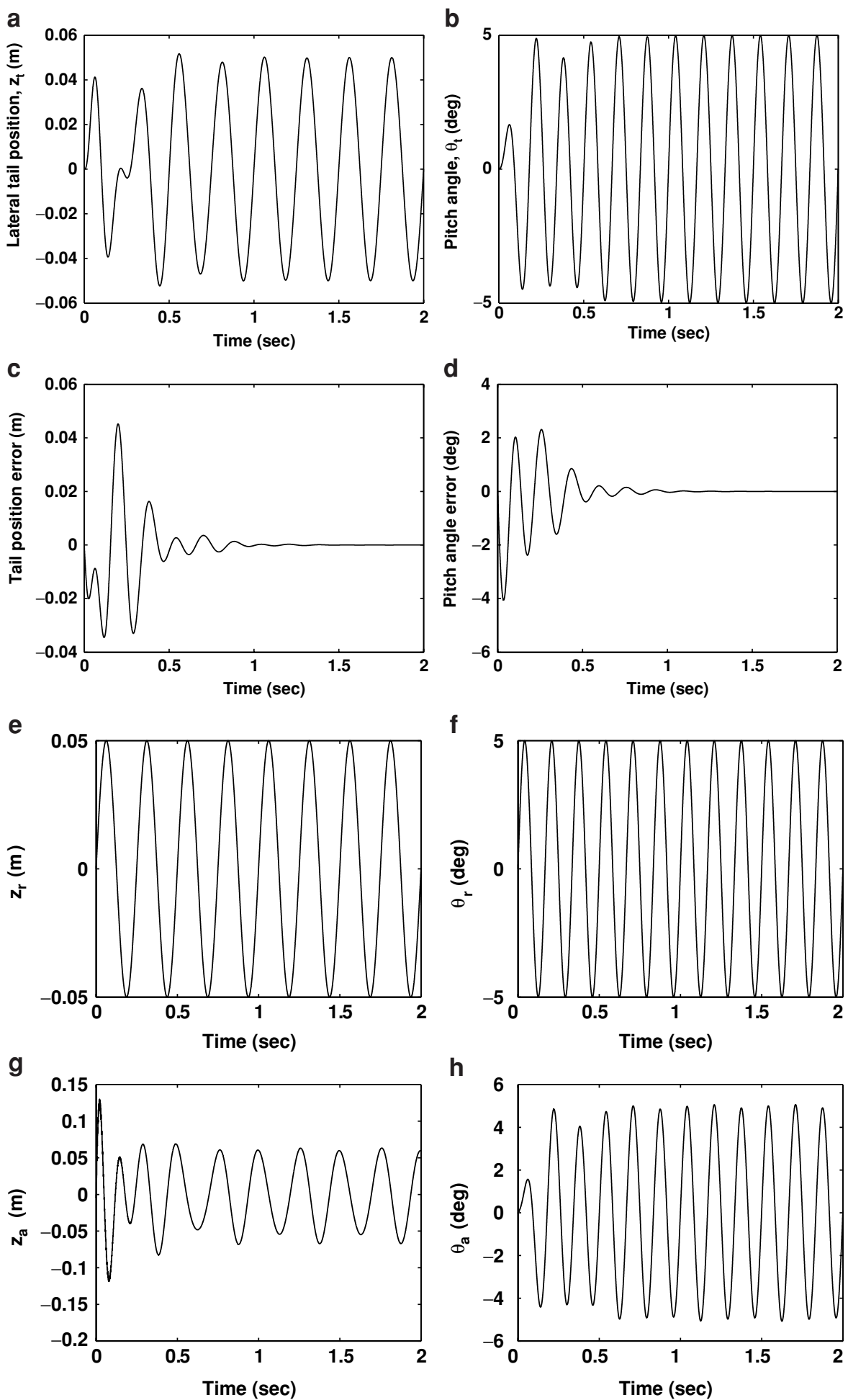

Figure 2 Oscillation control: $\omega_{z}=4, \omega_{\theta}=6 \mathrm{rad} / \mathrm{s}$. (a) Lateral position, $z_{\mathrm{t}}(\mathrm{m})$, (b) pitch angle, $\theta_{\mathrm{t}}\left({ }^{\circ}\right)$, (c) tail position error, $z_{\mathrm{t}}-z_{\mathrm{r}}(\mathrm{m}),(\mathrm{d})$ pitch angle error, $\theta_{\mathrm{t}}-\theta_{\mathrm{r}}\left({ }^{\circ}\right)$, (e) reference trajectory, $z_{\mathrm{r}}(\mathrm{m}),(\mathrm{f})$ reference trajectory, $\theta_{\mathrm{r}}\left({ }^{\circ}\right)$, (g) control input, $z_{\mathrm{a}}(\mathrm{m}),(\mathrm{h})$ control input, $\theta_{\mathrm{a}}\left(^{\circ}\right)$, (i) state, $x_{\mathrm{f}_{1}},(\mathrm{j})$ state, $x_{\mathrm{f}_{2}},(\mathrm{k})$ state, $x_{\mathrm{f}_{3}}$.

\section{SIMULATION RESULTS}

This section presents the results of digital simulation. The hydrodynamic parameters are taken from Harper et al (1998) and are collected in the appendix. For an illustration, reference trajectories of the form $y_{\mathrm{r}}(t)=$ $\left[A_{z} \sin \left(\omega_{z} t\right), A_{\theta} \sin \left(\omega_{\theta} t\right)\right]^{\mathrm{T}}$ are selected, where $A_{z}=$ $0.05 \mathrm{~m}$ and $A_{\theta}=5^{\circ}$. For the given $y_{\mathrm{r}}, \Pi_{e}(s)=\left(s^{2}+\right.$ $\left.\omega_{z}^{2}\right)\left(s^{2}+\omega_{\theta}^{2}\right)$, and one has $x_{\mathrm{s} z}, x_{\mathrm{s} \theta} \in R^{4}$. The augmented matrix $A_{\mathrm{a}}$ has seven stable and eight imaginary 
i

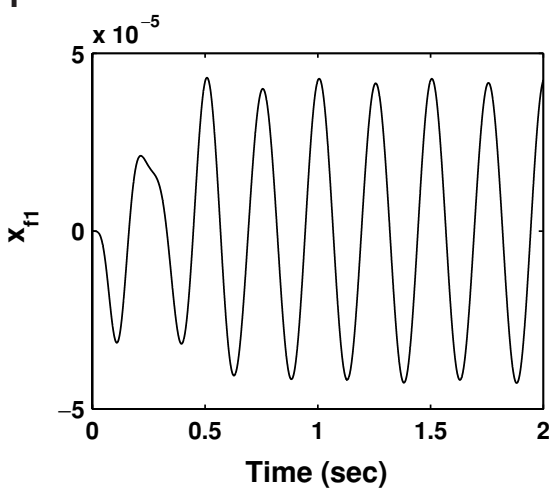

k

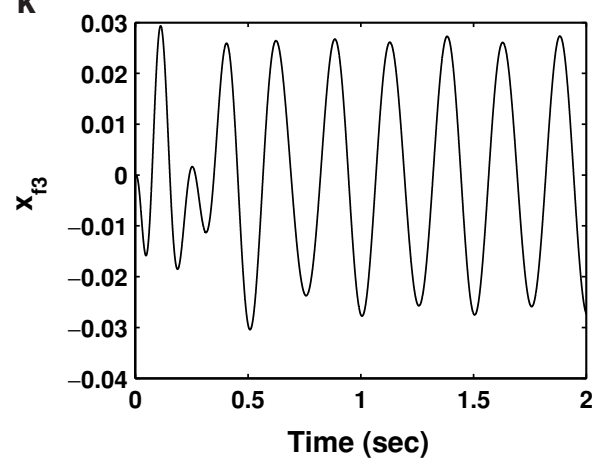

j

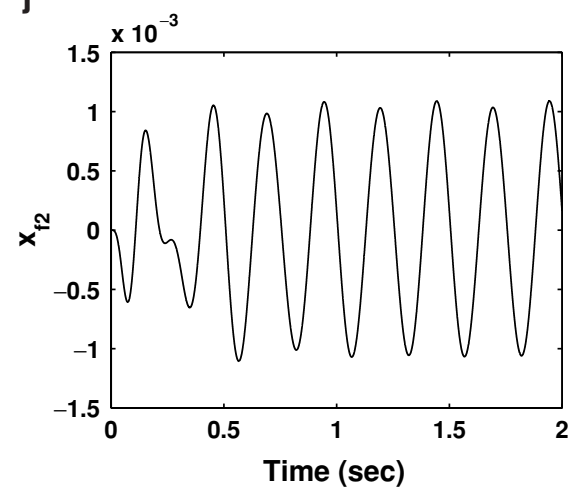

Figure 2 (Continued).

eigenvalues, and it is necessary to introduce feedback for the stabilization of the marginally stable matrix $A_{\mathrm{a}}$. The feedback matrices $K_{\mathrm{a}}$ and $F$ are determined using pole placement technique. These poles for simulation have been obtained by trial and error and by observing simulated responses. For simulation, initial conditions are assumed to be zero.

It is desired to control the foil so that the lateral displacement and pitch angle trajectories oscillate with distinct frequencies, where $\omega_{z}=4$ and $\omega_{\theta}=6 \mathrm{rad} / \mathrm{s}$. Simulated responses are shown in Figure 2. It is seen that the output vector $\left(z_{\mathrm{t}}(t), \theta_{\mathrm{t}}(t)^{\mathrm{T}}\right.$ smoothly converges to the reference trajectory $y_{\mathrm{r}}(t)$. After the initial transient, which is of the order of $1 \mathrm{~s}$, the lateral displacement and pitch angle trajectories are sinusoidal functions of time and have specified magnitudes and frequencies. The maximum tracking errors for the lateral and rotational motion are $5 \mathrm{~cm}$ and $6^{\circ}$, respectively. The control inputs $z_{\mathrm{a}}$ and $\theta_{\mathrm{a}}$ have peak magnitudes $14 \mathrm{~cm}$ and $5^{\circ}$, respectively. The filter state variables associated with the Theodorsen function are sinusoidal in the steady state. Simulation is also done for $\omega_{1}=6 \mathrm{rad} / \mathrm{s}$ and $\omega_{2}=4 \mathrm{rad} / \mathrm{s}$ as well as for identical frequencies. Sinusoidal trajectory tracking is smoothly accomplished in each case.

\section{CONCLUSIONS}

This paper has considered the control of oscillations of a 2-D hydrofoil for the purpose of producing maneuvering and propulsive forces. Two springs for transmitting forces to the foil have been used, which are like the tail tendons of fishes. Based on the servomechanism theory, a control law has been derived for the asymptotic pitch and plunge oscillatory trajectory control. Simulation results showed that in the closed-loop system the plunge and pitch can precisely follow sinusoidal trajectories of distinct magnitudes and frequencies.

\section{APPENDIX}

\section{System parameters}

The system parameters for simulation have been taken from Harper et al (1998).

$$
\begin{array}{ll}
a=0.02 \mathrm{~m} & b=0.02 \mathrm{~m} \\
U=0.5 \mathrm{~m} / \mathrm{s} & \rho=1000 \mathrm{Kg} / \mathrm{m}^{3} \\
K_{z}=124 \mathrm{~N} / \mathrm{m}^{2} & K_{\mathrm{t}}=10000 \mathrm{~N} / \mathrm{rad} \\
m=0 & \mathcal{F}=0
\end{array}
$$

\section{REFERENCES}

Azuma A. 1992. The bio-kinetics of flying and swimming. New York: Springer-Verlag.

Sfakiotakis M, Lane DM, Davies JBC. 1999. Review of fish swimming modes for aquatic locomotion. IEEE 7 Oceanic Engg, 24:237-52.

Bandyopadhyay PR, Castano JM, Dick J. 1999.

Biologically-inspired bodies under surface waves-Part 1: Load measurement. ASME $\mathcal{F}$ Fluids Eng, 121:469-78. 
Bandyopadhyay PR, Singh SN, Chockalingam F. 1999.

Biologically-inspired bodies under surface waves-part 2: Theoretical control of maneuvering, ASME $\mathcal{F}$ Fluids Eng, 121:479-87.

Triantafyllou GS, Triantafyllou MS. 1995. An efficient swimming machine. Sci Amer, 272:64-70.

Kato N. 2002. Pectoral fin controllers. In: Ayers J, Davis JL, Rudolph A (Eds), Neurotechnology for Biometric Robots, Cambridge: MIT Press.

Harper KA, Berkemeier MD, Grace S. 1998. Modeling the dynamics of spring-driven oscillating-foil propulsion. IEEE $\mathcal{F}$. Oceanic Eng, 23(3):285-96.

Lee J-S, Kim C, Rho O-H. 2003. The modification of airfoil shape for optimal aerodynamic performance on flapping-airfoil in low-Reynolds number flow. 41st Aerospace Sciences Meeting \& Exhibit, Reno, Nevada, AIAA 421.

Lauder GV, Drucker EG. 2004. Morphology and experimental hydrodynamics of fish fin control surfaces. IEEE 7 Oceanic Eng, 29:556-71.

Walker JA. 2004. Kinematics and performance of maneuvering control surfaces in teleost fishes. IEEE $\mathcal{7}$ Oceanic Eng, 29:572-84.
Bandyopadhyay PR. 2002. Maneuvering hydrodynamics of fish and small underwater vehicles. Integ Comp Biol, 42:102-17.

Triantafyllou MS, Techet AH, Hover FS. 2004. Review of experimental work in biomimetic foils. IEEE $\mathcal{F}$ Oceanic Eng, 29:585-94.

Singh SN, Simha A, Mittal R. 2004. Biorobotic AUV maneuvering by pectoral fins: inverse control design based on CFD parameterization. IEEE $\mathcal{7}$ Oceanic Eng, 29:777-85.

Mittal R. 2004. Computational modeling in biohydrodynamics: Trends, challenges, and recent advances. IEEE $\mathcal{F}$ Oceanic Eng, 29:595-604.

Lighthill MJ. 1970. Aquatic animal propulsion of high electromechanical efficiency. 7 Fluid Mech, 44:265-301.

Theodorsen T. 1935. General theory of aerodynamic instability and the mechanism of flutter. Tech Report 496, NACA.

Davison EJ. 1976. The robust control of a servomechanism problem for linear time-invariant multivariable systems. IEEE Trans Autom Control 21:25-34.

Wonham WM. 1985. Linear multivariable control: Geometric approach, 3rd ed. New York: Springer-Verlag. 

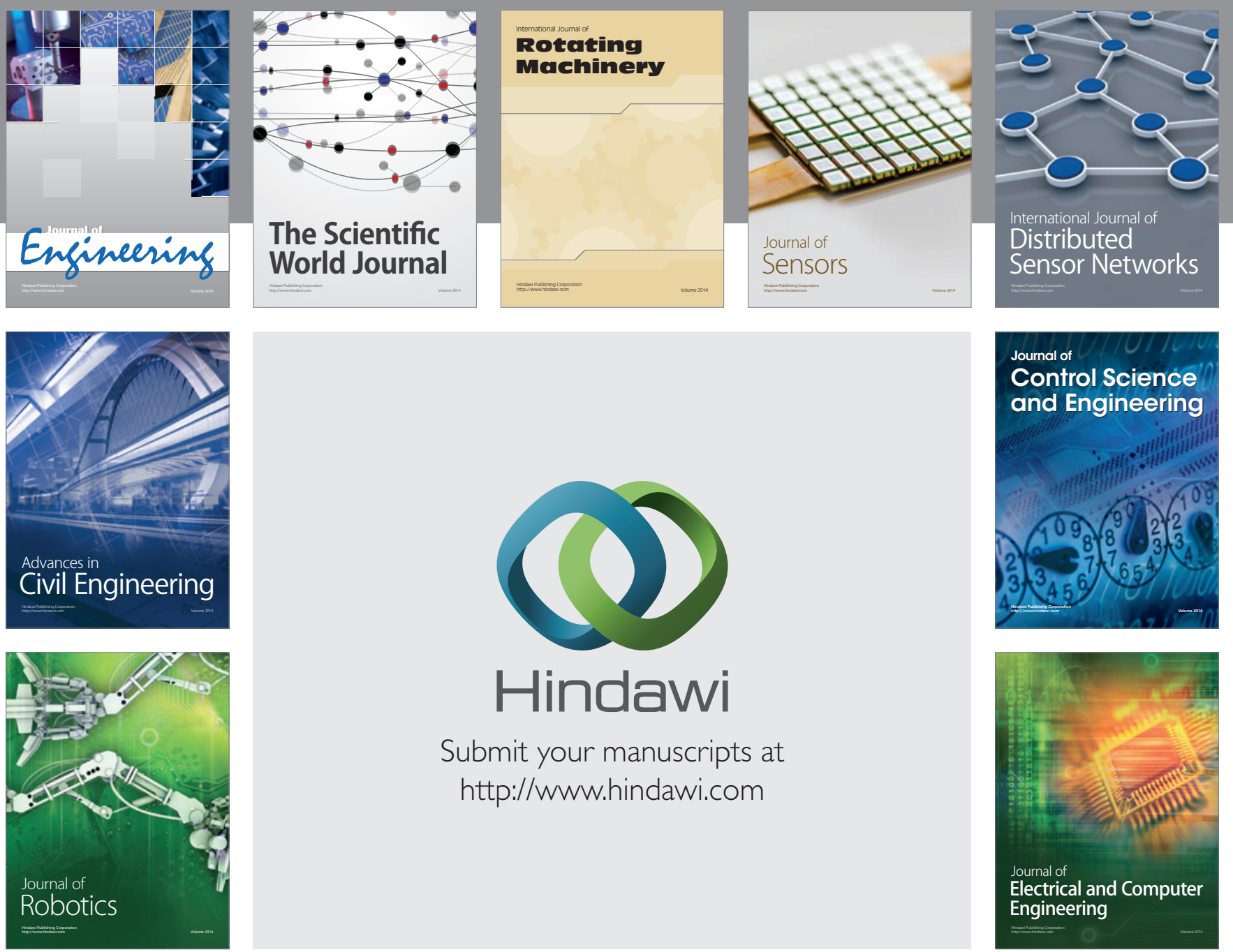

Submit your manuscripts at

http://www.hindawi.com
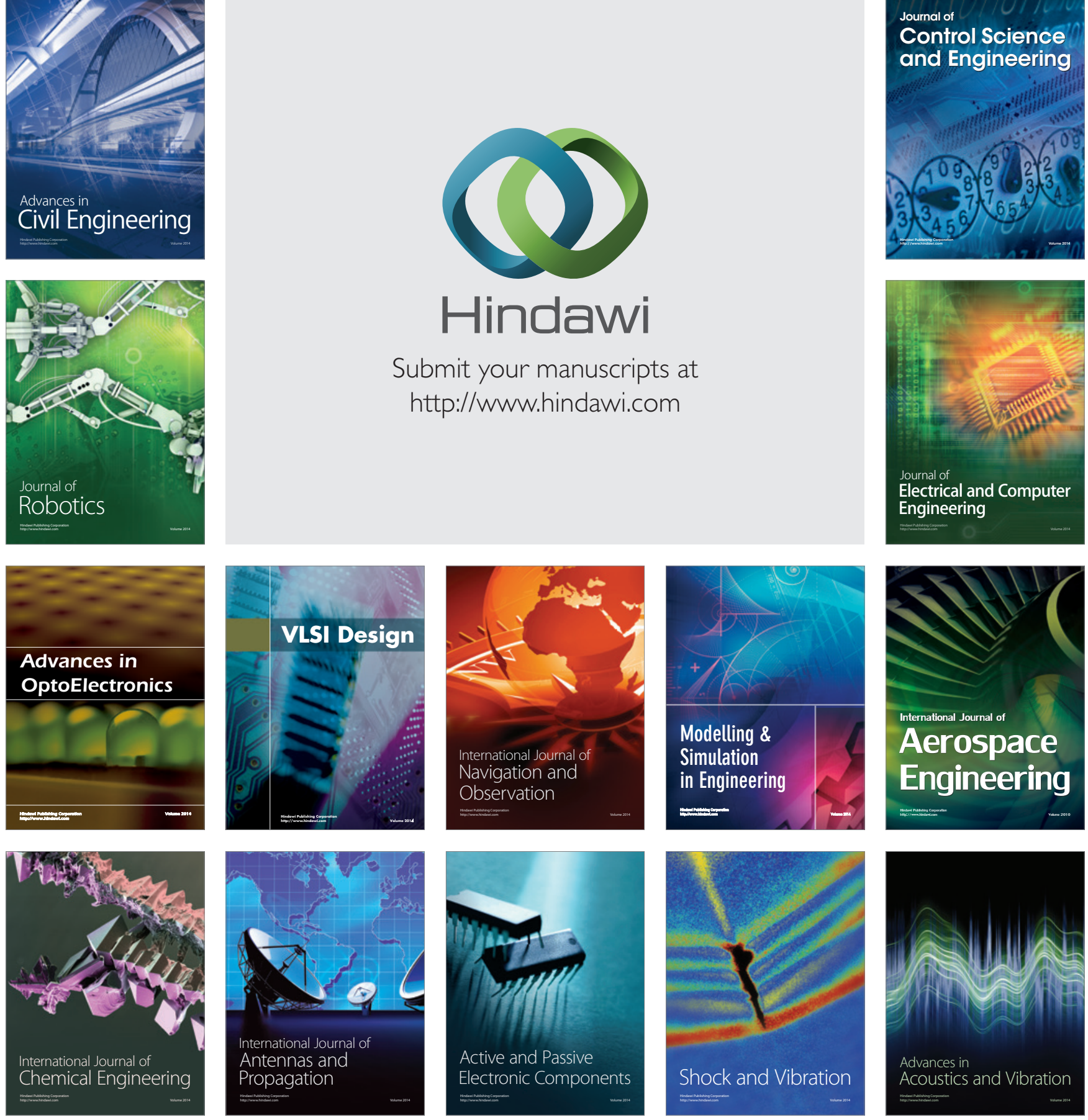